\title{
Application of Nuclear Emulsion to Neutron Emission Profile Diagnostics in the National Spherical Torus Experiment*)
}

\author{
Mitsutaka ISOBE ${ }^{1,2)}$, Douglass S. DARROW ${ }^{3)}$, Alvin L. ROQUEMORE ${ }^{3)}$, Kunihiro MORISHIMA ${ }^{4)}$, \\ Hideki TOMITA $^{4)}$, Haruna MINATO ${ }^{4)}$ and Kunihoro OGAWA ${ }^{1)}$ \\ 1) National Institute for Fusion Science, 322-6 Oroshi-cho, Toki 509-5292, Japan \\ ${ }^{2)}$ The Graduate University for Advanced Studies, 322-6 Oroshi-cho, Toki 509-5292, Japan \\ 3) Princeton Plasma Physics Laboratory, Princeton, New Jersey 08543-0451, USA \\ ${ }^{4)}$ Nagoya University, Furo-cho, Chikusa-ku, Nagoya 464-8603, Japan
}

(Received 21 November 2012 / Accepted 24 February 2013)

\begin{abstract}
The technology for OPERA experiments in neutrino physics was applied to neutral-beam-heated deuterium discharges of NSTX in order to measure d-d neutron emission profile. The diagnostic system consisted of nuclear emulsions named OPERA films and the automatic track scanning system S-UTS developed in Nagoya University. A neutron collimator having three channels was temporarily built for this purpose. The nuclear emulsion indicated peaked neutron emission profiles at the plasma center in NSTX as expected.
\end{abstract}

(c) 2013 The Japan Society of Plasma Science and Nuclear Fusion Research

Keywords: neutron, nuclear emulsion, NBI, NSTX

DOI: $10.1585 /$ pfr.8.2402068

\section{Introduction}

Intense heating by high-energy neutral beam (NB) injection is regularly performed in existing magnetically confined fusion plasma experiments to explore attainable range of high $-\beta$ value. In such a heating scheme, most of the fusion neutrons emitted from the plasma are produced by so-called beam-plasma reactions. In other words, the neutron emission rate is closely related to the slowing down and/or confinement of NB-injected energetic ions. Therefore, measurement of a spatially resolved neutron rate can provide a profile of energetic beam ions. This makes a contribution to not only the assessment of fundamental parameters such as fusion power emission profiles and/or NB energy deposition but also the study of the anomalous transport of energetic ions caused by Alfvénic modes and turbulences. For this reason, large tokamaks such as the Joint European Torus (JET), the Tokamak Test Fusion Reactor (TFTR), and the JAERI Tokamak-60 Upgrade (JT-60U) have been equipped with full-scale neutron profile diagnostic systems consisting of fast-neutron scintillation detector arrays and huge multi-channel collimators [1-5]. In retrospect, the first measurement of neutron emission profile was carried out by Strachan et al. in the Princeton Large Torus (PLT) by using nuclear emulsions [6]. It was experimentally revealed that the neutron emission profile in an NB-heated plasma is broader than that in an ohmic plasma. The nuclear emulsion was also applied to the Axisymmetric Divertor Experiment (ASDEX) to measure neutron profiles and energy spectra [7]. The

author'se-mail: isobe@nifs.ac.jp

*) This article is based on the presentation at the 22nd International Toki Conference (ITC22). nuclear emulsion is classified into a track detector. Fast neutrons emitted from fusion plasmas produce recoil proton tracks inside the emulsion. Nuclear emulsion can in principle work simultaneously as a neutron camera and as an energy spectrometer if the track length and scatter angle of recoil protons are determined. In the past, recoil proton tracks were read and/or counted through visual inspection using a microscope, which was a laborious task. Recently, an excellent automatic track scanning system with continuous three-dimensional tomographic image read-out, called the Super-Ultra Track Selector (S-UTS) [8], was developed in the F-laboratory, Graduate School of Science, Nagoya University for the neutrino experiment called OPERA. Because of this background, feasibility and capability studies of nuclear emulsion for nuclear fusion were initiated in the collaboration between the National Institute for Fusion Science (NIFS) and Nagoya University $[9,10]$. In this study, nuclear emulsions called OPERA film [11] were applied to the National Spherical Torus Experiment (NSTX) at the Princeton Plasma Physics Laboratory to try measuring spatial neutron yields with the help of S-UTS. An implementation of a tangential neutron profile monitor was considered in the NSTX about 10 years ago $[12,13]$. Although neutron collimators were preliminarily designed on that occasion, the monitor has not yet been realized.

\section{Experimental Setup}

\subsection{NSTX as a neutron source}

The NSTX is a low aspect ratio spherical torus operating at the mega ampere level [14]. It is normally operated with deuterium gases, and consequently $\mathrm{d}-\mathrm{d}$ neutrons are 


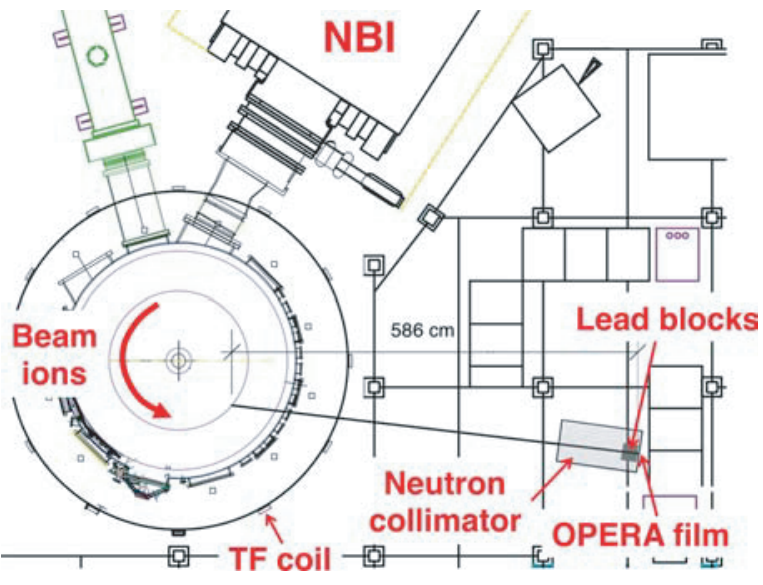

Fig. 1 Configuration of the temporary neutron collimator system installed from February 28, 2008 to March 6, 2008 for the test irradiation of nuclear emulsion on NSTX.

produced in NSTX plasmas. An intense NB injector consisting of three ion sources is installed on NSTX, injecting fairly high-energy beam ions tangentially in the counterclockwise direction (as seen from the top) with an injection energy $E_{\mathrm{b}} /$ power $P_{\mathrm{nb}}$ of $90 \mathrm{keV} / 7 \mathrm{MW}$. The fuel-ion temperature was typically equal to or around $1 \mathrm{keV}$. The energetic beam ions play a dominant role in producing fusion neutrons. The total neutron emission rate measured with calibrated ${ }^{235} \mathrm{U}$ fission chambers goes up to $5 \times 10^{14}(\mathrm{n} / \mathrm{s})$ in beam-heated NSTX plasmas.

\subsection{Neutron collimator}

Configuration of the neutron collimator system on NSTX is schematically depicted in Fig. 1. Three channel collimators were prepared temporarily for these experiments and were made from a stack of borated polyethylene blocks. Lead blocks were partially used near the irradiation point to reduce contamination due to $\gamma$-rays. Three unistruts made of aluminum were mounted inside the polyethylene stack as a path for the fast neutrons, having a foursquare opening of $2 \mathrm{~cm} \times 2 \mathrm{~cm}$ and length $l$ of $\sim 1 \mathrm{~m}$. The collimators were placed vertically from the equatorial plane in a downward direction, i.e., $Z=0 \mathrm{~cm}$, $-10 \mathrm{~cm}$, and $-20 \mathrm{~cm}$. Each line of sight was aimed at the space between two adjacent toroidal field (TF) coils to avoid scattering effects of the fast neutrons. Nuclear emulsions were set on the collimator end that was about $6 \mathrm{~m}$ away from the plasma axis.

\subsection{Nuclear emulsion}

The nuclear emulsion called OPERA film was applied to NSTX, functioning in principle as a neutron energy spectrometer. The fundamentals of neutron detection by means of nuclear emulsion are as follows. The energy of a recoil proton $E_{\mathrm{p}}$ generated by a fast neutron with energy $E_{\mathrm{n}}$ can be expressed as $E_{\mathrm{p}}=E_{\mathrm{n}} \cdot \cos ^{2} \theta$. Here, $\theta$ is the angle between the direction of the scattered neutron and the

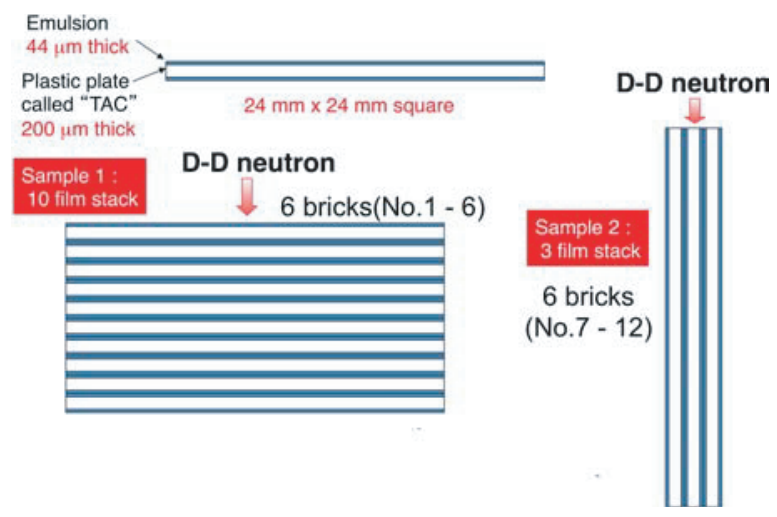

Fig. 2 Structure of OPERA film irradiated at NSTX. Emulsion layers with a thickness of $44 \mu \mathrm{m}$ were created on both side of the plastic base.

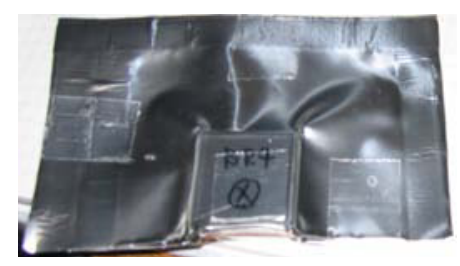

Fig. 3 External appearance of an OPERA film used in the NSTX. The emulsion is vacuum-packed using a thin aluminum sheet.

direction of the recoil proton. The direction of the recoil protons with maximum energy $(\theta=0)$ is the same as the direction of the incident fast neutrons. For recoil protons generated by $d-d$ neutrons, the maximum track length is expected to be about $40 \mu \mathrm{m}$ inside the emulsion. The structure of the nuclear emulsion used in NSTX is schematically depicted in Fig. 2. Two different types of films, i.e., 6 bricks of 10 film stacks and 6 bricks of 3 film stacks, were used in NSTX. Emulsion layers with a thickness of $44 \mu \mathrm{m}$ were created on both sides of the plastic base. Because each layer was unfortunately not thick enough unfortunately to obtain a neutron energy spectrum, in this study the number of tracks due to recoil protons were counted. After irradiation at NSTX, the emulsion was sent back to Japan and recoil proton tracks were counted by using the S-UTS at Nagoya University. The absolute neutron detection efficiency of each film was about $10^{-3}$. The external appearance of an OPERA film used in this experiment is shown in Fig. 3.

\section{Results}

Irradiation was performed during two continuous days of experiment (February 29, 2008 and March 3, 2008) in NB-heated plasmas of NSTX. Two photographs of nuclear emulsions from 10 film stacks are shown in Fig. 4. An emulsion without irradiation as a reference and an example of tracks recorded in irradiated emulsion are shown in Figs. 4 a) and 4 b), respectively. Fine ionizing tracks are 


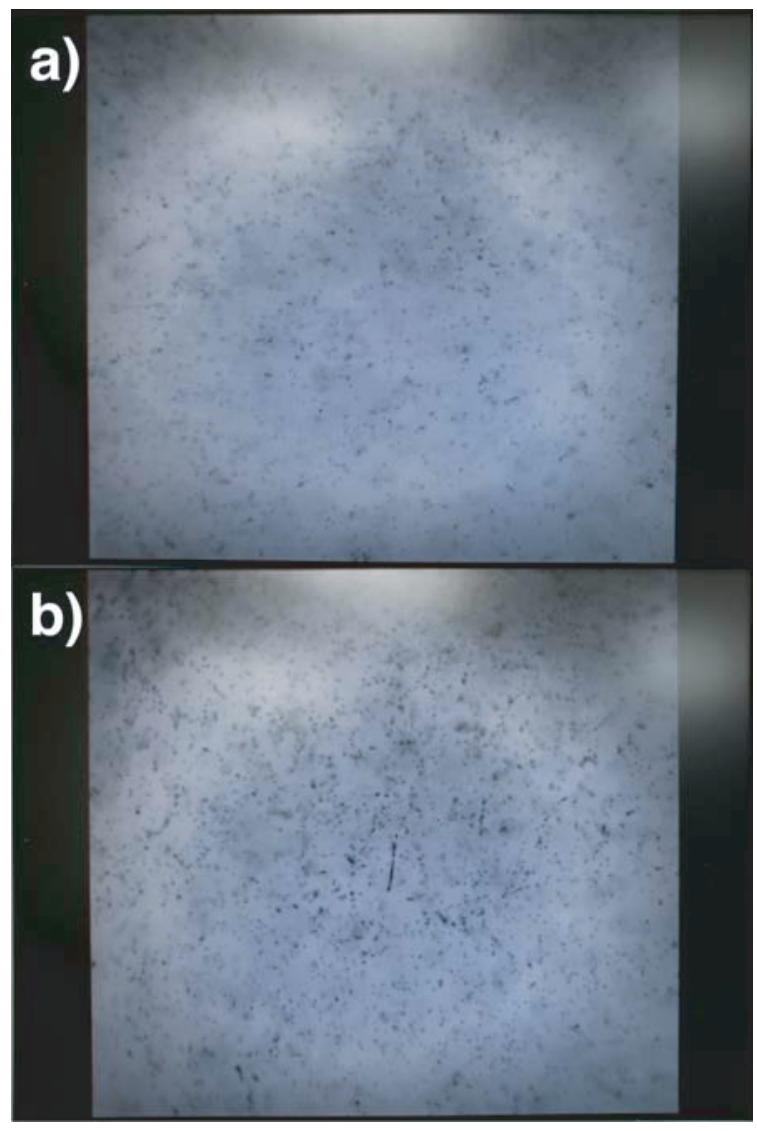

Fig. 4 Photographs showing tracks recorded in the nuclear emulsion. a) Non-irradiated nuclear emulsion (BR06) at NSTX as a reference. b) Irradiated emulsion (BR01). Thick tracks owing to recoil protons are seen in b).

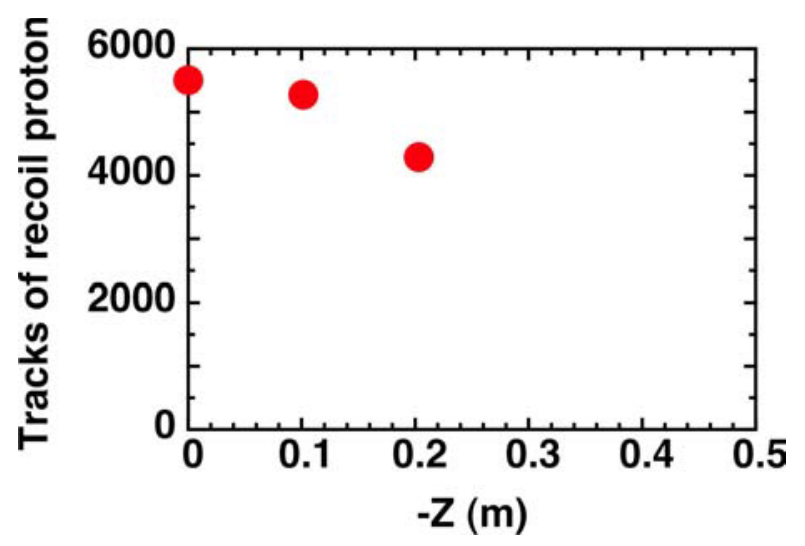

Fig. 5 Number of recoil proton tracks recorded in the nuclear emulsion as a function of $\mathrm{Z}$. This is equivalent to a d-d neutron profile, i.e., beam ion profile in NSTX.

observed in Fig. 4 a), which result from electrons due to $\gamma(\mathrm{X})$-rays. On the other hand, in addition to fine tracks, thick tracks can be recognized in Fig. 4 b), which are produced owing to recoil protons kicked by fast d-d neutrons.

The number of recoil proton tracks as a function of $Z$ is shown in Fig. 5. The S-UTS indicates that the highest number of recoil proton tracks was recorded in the nuclear emulsion placed at the equatorial position and the number of tracks tended to decrease along the downward direction. This observation suggests us that the profile of the energetic beam ions in NSTX is certainly peaked at the plasma center as expected.

\section{Summary}

In summary, the technology for neutrino experiments consisting of nuclear emulsions called OPERA films and the superior automatic track scanning system called SUTS was applied to beam-heated deuterium discharges of the NSTX in order to obtain radial profiles of beam ions through the measurement of a profile of a $\mathrm{d}-\mathrm{d}$ neutron emission. The experiment indicated that the beam ion profile in the NSTX is peaked at the plasma center as expected. Nuclear emulsion is attractive as a tool for neutron diagnostics in fusion not only for the compactness but also for its capability of providing neutron profiles and energy spectra. It can also clearly distinguish between neutron events and $\gamma(\mathrm{X})$-ray events. Further performance checks of nuclear emulsion as a tool for neutron diagnostics are being performed in deuterium discharges of the Korea Superconducting Tokamak Advanced Research (KSTAR).

\section{Acknowledgments}

The authors would like to thank the NSTX team for making this work possible, as well as Dr. Mamiko Sasao of Doshisya University for fruitful discussion about the role of nuclear emulsion in magnetically confined fusion plasma experiments. This work was supported by a Japan/U.S. Cooperation in Fusion Research and Development, and NIFS Collaboration Research Program (Contact No. NIFS08KOBH001 and No. NIFS12KOAH029)

[1] J.M. Adams et al., 14th European Conference on Controlled Fusion and Plasma Physics, Madrid, June 22-26, 1987, Vol. 11D, Part III, p.1224.

[2] J.M. Adams et al., Nucl. Instrum. Methods Phys. Res. A329, 277 (1993).

[3] H. Hendel et al., Rev. Sci. Instrum. 56, 1081 (1985).

[4] A.L. Roquemore et al., Rev. Sci. Instrum. 61, 3163 (1990).

[5] M. Ishikawa et al., Rev. Sci. Instrum. 73, 4237 (2002).

[6] J.D. Strachan et al., Phys. Lett. 66A, 295 (1978).

[7] B. Wolle et al., Nucl. Instrum. Methods Phys. Res. A368, 425 (1996).

[8] K. Morishima and T. Nakano, J. Instrumentation 5, P04011 (2010).

[9] Y. Nomura et al., Plasma Fusion Res. 6, 2402148 (2011).

[10] H. Minato et al., this conference.

[11] T. Nakamura et al., Nucl. Instrum. Methods Phys. Res. A556, 80 (2006).

[12] A.L. Roquemore et al., 30th EPS Conference on Contr. Fusion and Plasma Phys., St. Petersburg, July 7-11, 2003 ECA Vol. 27A, P-4.75.

[13] A.L. Roquemore et al., 8th IAEA Technical Meeting on Energetic Particles in Magnetic Confinement Systems, San Diego, CA, October 6-8, 2003, P4.

[14] M. Ono et al., Nucl. Fusion 40, 557 (2000). 